Journal Club

Editor's Note: These short, critical reviews of recent papers in the Journal, written exclusively by graduate students or postdoctoral fellows, are intended to summarize the important findings of the paper and provide additional insight and commentary. For more information on the format and purpose of the Journal Club, please see http://www.jneurosci.org/misc/ifa_features.shtml.

\title{
Can Cortical Stimulation of Inferior Frontal Cortex Enhance Proactive Control?
}

\author{
Vanessa M. Johnen, ${ }^{1,2}$ Ethan R. Buch, ${ }^{3,4}$ and Franz-Xaver Neubert ${ }^{1,2}$ \\ ${ }^{1}$ Department of Experimental Psychology, University of Oxford, Oxford OX1 3UD, United Kingdom, ${ }^{2}$ Centre for Functional Magnetic Resonance Imaging of \\ the Brain, University of Oxford, John Radcliffe Hospital, Oxford OX3 9DU, United Kingdom, ${ }^{3}$ Human Cortical Physiology and Neurorehabilitation Section, \\ National Institute of Neurological Disorders and Stroke, National Institutes of Health, Bethesda, Maryland 20814, and ${ }^{4}$ Center for Neuroscience and \\ Regenerative Medicine, Uniformed Services University of Health Sciences, Bethesda, Maryland 20814 \\ Review of Wessel et al.
}

Brain stimulation is an important tool in neuroscience. It helps localize functions to specific cortical areas, provides insight into how information is organized in the cerebral cortex, and has long been explored for clinical therapeutic uses. For example, transcranial magnetic stimulation (TMS) to the prefrontal cortex is used in the treatment of acute depression (Kozel and George, 2002). In another study, direct electrical stimulation (DES) over part of the primary motor cortex that controls hand and wrist movements was used in a randomized clinical trial to facilitate rehabilitation for recovery of upper limb motor function in stroke patients (Harvey and Winstein, 2009). Nevertheless, perhaps the greatest therapeutic efficacy has been observed with deep brain stimulation of subcortical structures in patients with severe neurological disorders (e.g., the subthalamic nucleus in Parkinson's disease; Williams and Okun, 2013). This is puzzling, since the function of the neocortex is better understood than many subcortical structures (cortex is more accessible for neurophysiological and neuroimaging experiments). Moreover, it is thought that effects of cortical stimula-

Received Feb. 11, 2014; revised March 13, 2014; accepted March 19, 2014. Correspondence should be addressed to Vanessa M. Johnen, Department of Experimental Psychology, University of Oxford, South Parks Road, Oxford 0X1 3UD, UK. E-mail: vanessa.johnen@psy.ox.ac.uk.

DOI:10.1523/JNEUROSCI.0590-14.2014

Copyright $\odot 2014$ the authors $\quad 0270-6474 / 14 / 346125-03 \$ 15.00 / 0$ tion would be more specific compared with subcortical stimulation and hence might promote desired effects and mitigate unwanted side effects.

A recent study used cortical stimulation to augment a very specific cognitive function-proactive inhibitory motor control, which is defined as the preparation to stop an upcoming response tendency in line with one's goals. Thus, "proactive stopping" serves an alternative control mechanism to "reactive stopping," where subjects stop a response outright once instructed by an external signal. This study is methodologically interesting as it advances the use of cortical DES for investigating cognitive function within task contexts. Specifically, it provides insight into the role of the inferior frontal cortex (IFC) in executive control of motor behavior. Moreover, it serves as an interesting example of how cortical stimulation can be used to manipulate neural processing and subsequently, behavior.

The primary aim of Wessel et al. (2013) was to investigate the involvement of right IFC (rIFC) in proactive, goaldriven inhibitory motor control. Four patients with intractable epilepsy were implanted with subdural electrocorticography (ECoG) arrays, and DES was applied over rIFC. The stimulation site was identified via a blinded functional localizer derived from a known ECoG "signature" (increase in beta- and/or gamma-band power during stopping) commonly observed in lateral frontal electrodes during a stop-signal task. On most trials (67\%), patients executed a cued response (Go-trial). On some trials (33\%), however, patients were instructed to stop the movement execution when a secondary auditory stimulus was presented (Stop-trial). This stop-signal task is also referred to as the "Maybe stop" (MS) condition. In the main experiment, patients performed blocks of MS trials and blocks of "No stop" (NS) trials where no stop-signal occurred. The authors used a computercontrolled, event-related stimulation procedure, which delivered pairs of DES to rIFC during Go-trials $50 \mathrm{~ms}$ (Patient 1) or $100 \mathrm{~ms}$ (Patients 2, 3, and 4) before their predicted response time. They compared reaction times (RTs) of trials with and without stimulation. In two patients, DES was delivered to rIFC and a temporal lobe control site in alternating blocks.

The authors show that rIFC DES slows down Go-trial RTs relative to Go-trials in which no stimulation was delivered. Furthermore, this effect was context-dependent: slowing was greater for MS versus NS Go-trials. There is ample evidence supporting the notion that rIFC is involved in reactive stopping (Aron, 2011), which is consistent with findings of the current study (i.e., slowing on NS Gotrials following stimulation). However, context-dependent slowing as induced by 
rIFC stimulation was augmented further in situations when motor caution was required and a response tendency had to be stopped to succeed on the given trial (MS trials). This context-dependency might therefore suggest a role for rIFC in "proactive stopping”. This notion was further supported by two additional findings: (1) DES of the inferior temporopolar cortex control site in one patient did not elicit slowing in the MS context, and (2) the magnitude of braking augmentation caused by rIFC stimulation was also statedependent, as it was negatively correlated with the amount of slowing observed both across and within patients. Intriguingly, DES applied to an anterior superior temporal sulcus (STS) control site in a second patient also resulted in context-dependent slowing of Go-trial RTs. Post hoc investigation of this effect revealed that the STS control site in this patient also displayed the stopping-related ECoG signature during the functional localizer task.

The authors propose that rIFC is part of an executive control network responsible for implementing motor caution, braking, and outright stopping. Functional anatomical studies have suggested however, that processing underlying these related cognitive functions may be localized to subregions of rIFC. Chikazoe and colleagues (2009) have proposed that inferior frontal junction (IFJ) and right posterior inferior frontal gyrus are differentially involved in stop preparation and execution, respectively. This notion of functional specificity within rIFC subregions may also be supported by the results reported by Wessel and colleagues (2013). In their study, two patients were stimulated in a more dorsal area of rIFC (closer to IFJ) and exhibited significant slowing in both MS and NS trials, whereas the other two were stimulated in ventral rIFC and showed slowing only in MS trials. Future research should further explore the question of whether functional specialization related to different aspects of executive control exists in IFC subregions.

The study by Wessel et al. (2013) shows causal involvement of rIFC in higher cognitive processes, such as "proactive control." This finding can be compared with the discovery that neurons in ventral premotor cortex (PMv), a subregion located within caudal IFC, are not only active when complex motor plans are executed (i.e., when subjects grasp a particular object), but also when targets of complex motor behavior (graspable objects) are presented and when subjects observe others perform the same motor behavior. Ac- tivity of $\mathrm{PMv}$ during action, actionobservation, and observation of objects whose features constrain the possible action space (commonly referred to as "action affordances"; Baber et al., 2014) has been linked to empathy, self-awareness, and language. In line with this complex profile of $\mathrm{PMv}$, a similar functional profile is being established for IFC_-involvement in inhibition of both action execution and action affordances. rIFC's involvement in proactive stopping in situations of higher uncertainty has been interpreted in the context of "predictive coding" (Neubert and Klein, 2010). The brain might constantly catalog statistical regularities in the environment to predict future events, thereby minimizing surprise and need for behavioral adjustments. Similar principles might apply to rIFC in relation to stopping.

The finding that DES of anterior STS also leads to slowing in one patient suggests that in some cases anterior STS may also contribute to motor braking. This result raises an interesting question: if proactive inhibition is performed by a network of regions, would DES of any neural site that shows a stopping-related ECoG signature augment braking? Using an ECoG-based functional localizer related to stopping presents an elegant means of defining stimulation sites. This approach could be useful for mapping the distributed network involved in proactive inhibitory control. Through the combination of ECoG and multisite DES, one might be able to identify influences of one node upon another, and how network dynamics relate to both normal and pathological behavior.

Wessel et al. (2013) used a novel methodological approach to manipulate cognitive control with DES. First, individual rIFG stimulation sites were identified based on functional localizers for inhibitory control. Second, a double-blinded stimulation design was used in which neither experimenter nor patient were aware of whether stimulation was applied or not, or which cortical site was being stimulated. Last, the inclusion of a negative control site in the temporal lobe is a substantial advance over previous work. Overall, these findings have significant implications for the design of future experimental and therapeutic cortical stimulation protocols that aim to manipulate higher level cognitive processing.

Noninvasive TMS protocols have been successfully used to induce frequencydependent and spike-timing-dependent plasticity. Application of repetitive TMS to primary motor cortex can have longterm effects on corticospinal excitability in a frequency-dependent manner (Siebner and Rothwell, 2003), and evidence is emerging that paired-associative TMS over interconnected cortical sites can induce directed, long-term changes in effective connectivity in line with principles of spike-timing-dependent plasticity (STDP; Buch et al., 2011). Alternatively, noninvasive transcranial direct current stimulation (tDCS) is believed to alter membrane potentials instead of inducing neuronal firing, thereby enhancing activity-dependent plasticity. It is plausible that DES can induce any of these different forms of plasticity with far better spatial and temporal resolution, and thus may have greater therapeutic potential in appropriate patient populations. Combining our current understanding of cortical plasticity with insight from the novel DES paradigm, one might think of using prolonged cortical DES to selectively induce plasticity over a single site and to monitor potential long-term changes in behavior. Using the present task paradigm, one could probe whether proactive stopping can be enhanced by plasticity induction so that changes to cognitive function remain following removal of the stimulation. Particularly, rather than only looking at stimulation-induced slowing in MS Go-trials, one might quantify the effectiveness of the stopping-process itself (or the manipulation thereof) when stopping is required (e.g., by looking at stopsignal RT).

A further extension of the above proposal would involve selective enhancement of effective connectivity within a targeted corticocortical pathway. This could be accomplished via paired DES stimulation of connected areas at intervals that could plausibly lead to STDP and modification of synaptic efficacy. Behavioral changes in response to plasticity induction by means of pairing electrical activity in the motor cortex have been shown in freely behaving primates (Jackson et al., 2006). The neural implant used in this experiment induced spikingactivity in response to intrinsic spikingactivity, and was implanted into the cerebral gray matter rather than subdurally. However, in the light that TMS can selectively modulate connectivity within a corticocortical pathway and that DES can enhance the causal influence of one brain area over another at a given instant, it is not unreasonable to think that this form of electrical stimulation may prove useful 
for therapeutic applications based on modulation of aberrant network activity.

\section{References}

Aron AR (2011) From reactive to proactive and selective control: developing a richer model for stopping inappropriate responses. Biol Psychiatry 69:e55-e68. CrossRef Medline

Baber C, Parekh M, Cengiz TG (2014) Tool use as distributed cognition: how tools help, hinder and define manual skill. Front Psychol 5:116. CrossRef Medline

Buch ER, Johnen VM, Nelissen N, O'Shea J, Rushworth MF (2011) Noninvasive associative plasticity induction in a corticocortical pathway of the human brain. J Neurosci 31:1766917679. CrossRef Medline
Chikazoe J, Jimura K, Asari T, Yamashita K, Morimoto H, Hirose S, Miyashita Y, Konishi S (2009) Functional dissociation in right inferior frontal cortex during performance of go/no-go task. Cereb Cortex 19: 146-152. CrossRef Medline

Harvey RL, Winstein CJ, Everest Trial Group (2009) Design for the Everest randomized trial of cortical stimulation and rehabilitation for arm function following stroke. Neurorehabil Neural Repair 23:32-44. CrossRef Medline

Jackson A, Mavoori J, Fetz EE (2006) Long-term motor cortex plasticity induced by an electronic neural implant. Nature 444:56-60. CrossRef Medline

Kozel FA, George MS (2002) Meta-analysis of left prefrontal repetitive transcranial magnetic stimulation (rTMS) to treat depression. J Psychiatr Pract 8:270-275. CrossRef Medline

Neubert FX, Klein MC (2010) What is driving inhibition-related activity in the frontal lobe? J Neurosci 30:4830-4832. CrossRef Medline

Siebner HR, Rothwell J (2003) Transcranial magnetic stimulation: new insights into representational cortical plasticity. Exp Brain Res 148:1-16. CrossRef Medline

Wessel JR, Conner CR, Aron AR, Tandon N (2013) Chronometric electrical stimulation of right inferior frontal cortex increases motor braking. J Neurosci 33:19611-19619. CrossRef Medline

Williams NR, Okun MS (2013) Deep brain stimulation (DBS) at the interface of neurology and psychiatry. J Clin Invest 123:45464556. CrossRef Medline 